\title{
THE GENERALIZED CONDITION NUMBERS OF BOUNDED LINEAR OPERATORS IN BANACH SPACES
}

\author{
GUOLIANG CHEN, YIMIN WEI and YIFENG XUE
}

(Received 1 May 2002; revised 3 March 2003)

Communicated by A. Pryde

\begin{abstract}
For any bounded linear operator $A$ in a Banach space, two generalized condition numbers $\bar{\kappa}(A)$ and $\hat{\kappa}(A)$ are defined in this paper. These condition numbers may be applied to the perturbation analysis for the solution of ill-posed differential equations and bounded linear operator equations in infinite dimensional Banach spaces. Different expressions for the two generalized condition numbers are discussed in this paper and applied to the perturbation analysis of the operator equation.
\end{abstract}

2000 Mathematics subject classification: primary 47A05, 65F20, $65 \mathrm{~J} 1$.

Keywords and phrases: Condition number, bounded linear operator, generalized inverse.

\section{Introduction}

The condition number of a matrix or operator plays important role in solving the linear equation and in computing the inverse of a matrix or operator. It also measures the distance from the given matrix to the set of singular matrices.

Let $X$ and $Y$ be Banach spaces, and $S$ be a closed subspace of $X$. Denote by $L(X, Y)$ the Banach space composed of all bounded linear operator from $X$ onto $Y$; $R(A)$ the range of $A ; N(A)$ the null space of $A ; S^{c}$ a topological complement of $S$ (for example, $X=S \oplus S^{c}$ ).

If $N(A)^{c}$ and ${\overline{R(A)^{c}}}^{c}$ are topological complements of closed subspaces $N(A)$ and $\overline{R(A)}$ (the closure of $R(A))$ in $X$ and $Y$ respectively, then $N(A)^{c}$ and $\overline{R(A)}^{c}$ are closed and

$$
X=N(A) \oplus N(A)^{c}, \quad Y=\overline{R(A)} \oplus \overline{R(A)^{c}} .
$$

(C) 2004 Australian Mathematical Society $1446-7887 / 04 \$ A 2.00+0.00$ 
A well-known result is that $N(A)$ (or $\overline{R(A)}$ ) has a topological complement $N(A)^{c}$ (or $\overline{R(A)}$ ) if and only if there exists a continuous projector $P$ (or $Q$ ) mapping $X$ onto $N(A)$ (or $R(A)$ ) (see [10]). Nashed proved that if $R(A)$ is closed and the following topological direct sums exists

$$
X=N(A) \oplus N(A)^{c}, \quad Y=R(A) \oplus R(A)^{c},
$$

then there exists a unique generalized inverse $A^{+}=A_{P, Q}^{+} \in L(Y, X)$ of $A$ (the subscripts $P$ and $Q$ imply that $A^{+}$depends on the projection operators $P$ and $Q$ ) such that

$$
\begin{aligned}
A A^{+} A & =A, & A^{+} A A^{+} & =A^{+}, \\
A^{+} A & =P_{N(A) c}, & A A^{+} & =P_{R(A)},
\end{aligned}
$$

where $P_{R(A)}$ and $P_{N(A)^{r}}$ are the continuous operators which project $Y$ and $X$ onto $R(A)$ along $R(A)^{c}$ and onto $N(A)^{c}$ along $N(A)$, respectively.

In the case when $A^{+}$exists, the generalized condition number is defined by

$$
\kappa^{+}(A)=\|A\|\left\|A^{+}\right\| .
$$

Many mathematicians discussed new definitions and properties of various condition numbers (see, for example, $[1,2,4,8,9,11]$ ).

With recent advances in numerical analysis in infinite dimensional Banach spaces, one needs to consider the condition number of a bounded linear operator. But in infinite dimensional Banach spaces, the condition number of a bounded linear operator is not always defined as in (1.3) because not all closed subspaces of a Banach space have topological complements (see [6]). So for some ill-posed differential equations in Banach spaces, there is no condition number (in the usual sense) associated with them.

In this paper, we first give an equivalent description of the reduced minimum modulus of bounded linear operators in Banach spaces. This description can be used to define the reduced minimum modulus of any element in a Banach algebra (see [12]). Then we will give two different definitions of the generalized condition numbers of bounded linear operators in Banach spaces which are more general and applicable than $\kappa^{+}(A)$ defined by (1.3). Using our definitions, we will establish the error estimate of the solution of the linear operator equation $A x=b$ via small perturbation $r$ on the right-hand side of the equation. We also give a comparison between two kinds of condition numbers.

\section{A generalized condition number}

In this section $A$ will be a bounded linear operator from $X$ onto $Y$. 
DEFINITION 2.1. Let $A \in L(X, Y)$. The reduced minimum modulus $r(A)$ of $A$ is defined by

$$
r(A)=\inf \{\|A x\|: \operatorname{dist}(x, N(A))=1, x \in X\},
$$

where $\operatorname{dist}(x, N(A))=\inf _{y \in N(A)}\|x-y\|$.

From (2.1), we get that for any $A \in L(X, Y)$,

$$
\|A x\| \geq r(A) \operatorname{dist}(x, N(A)), \quad \forall x \in X .
$$

Let $X^{*}$ and $Y^{*}$ be dual spaces of $X$ and $Y$ respectively, then for $A \in L(X, Y)$, we have $A^{*} \in L\left(Y^{*}, X^{*}\right)$. Moreover, $r(T)=r\left(T^{*}\right)$ and $R(A)$ is closed if and only if $r(A)>0$ (see, for example, [7, Theorem 5.13, Theorem 5.2]).

The following gives our definition of the generalized condition number.

DEFINITION 2.2. If $A \in L(X, Y)$ and $R(A)$ is closed, then the generalized condition number (or GCN) of $A$ is defined by

$$
\bar{\kappa}(A)=\|A\| r(A)^{-1} .
$$

REMARK. We should notice the following special cases.

(1) If $A \in L(X, Y)$ with $R(A)$ closed, then $\bar{\kappa}(A)=\bar{\kappa}\left(A^{*}\right)$.

(2) If $A \in L(X, X)$ is invertible, then $\bar{\kappa}(A)=\|A\|\left\|A^{-1}\right\|=\kappa(A)$.

(3) Let $A \in L(X, Y)$ with a generalized inverse $A^{+}$. Then by [3, Lemma 2.1],

so that

$$
\frac{1}{\left\|A^{+}\right\|} \leq r(A) \leq \frac{\left\|A^{+} A\right\|\left\|A A^{+}\right\|}{\left\|A^{+}\right\|}
$$

$$
\frac{\kappa^{+}(A)}{\left\|A^{+} A\right\|\left\|A A^{+}\right\|} \leq \bar{\kappa}(A) \leq \kappa^{+}(A)
$$

This indicates that if $X, Y$ are Hilbert spaces and $A^{+}$is the Moore-Penrose inverse of $A$, then $\bar{\kappa}(A)=\kappa^{+}(A)$.

(4) Let $A$ be an $m$-by- $n$ matrix, which can be regarded as a linear transformation from $C^{n}$ to $C^{m}$. Suppose further that $C^{n}$ and $C^{m}$ have norms, both of which are denoted by $\|\cdot\|$. We denote the dual norms by $\|\cdot\|_{D}$. Then the condition number

$$
\kappa(A)=\|A\| \alpha(A)^{-1}
$$

defined in [4], where $\alpha(A)=\min \left(\left\|A^{*} x\right\|_{D}:\|x\|_{D}=1, x \in R(A)\right\}$, coincides with $\bar{\kappa}(A)$ defined by (2.2). Because $N\left(A^{*}\right)^{\perp}=R(A)$ and any $x \in C^{m}$ can be decomposed as $x=x_{1}+x_{2}$, where $x_{1} \in N\left(A^{*}\right), x_{2} \in R(A)$, we have $\alpha(A)=r\left(A^{*}\right)=r(A)$. 
Now we present an equivalent description of $r(A)$ as follows:

THEOREM 2.3. If $A \in L(X, Y)$ and $R(A)$ is closed, then

$$
r(A)=\inf \left\{\|A-B\|: \overline{R(B)} \subset_{\neq} R(A), N(B) \supset N(A), B \in L(X, Y)\right\} .
$$

PROOF. We will use some concepts and theorems from Taylor's book (see [10]) while proving this theorem. The proof is divided into two parts: (i) $N(A)=0$, and (ii) $N(A) \neq 0$.

(i) Suppose that $N(A)=0$, then $N(B) \supset N(A)$. From

$$
\overline{R(B)} \subset_{\neq} R(A), \quad N\left(A^{*}\right)=\overline{R(A)}^{\perp}, \quad \text { and } \quad N\left(B^{*}\right)=\overline{R(B)}^{\perp},
$$

where $\overline{R(A)}{ }^{\perp}=\left\{f \in Y^{*}: f(y)=0, \forall y \in \overline{R(A)}\right\}$, we have $N\left(A^{*}\right) \subset_{\neq} N\left(B^{*}\right)$.

Let $x^{*} \in N\left(B^{*}\right)$ such that $\operatorname{dist}\left(x^{*}, N\left(A^{*}\right)\right)=1$. Then, for $y^{*} \in N\left(A^{*}\right)$, we have

$$
\begin{aligned}
r(A)=r\left(A^{*}\right) & \leq\left\|A^{*} x^{*}\right\|=\left\|\left(A^{*}-B^{*}\right)\left(x^{*}-y^{*}\right)\right\| \\
& \leq\left\|A^{*}-B^{*}\right\|\left\|x^{*}-y^{*}\right\|=\|A-B\|\left\|x^{*}-y^{*}\right\|,
\end{aligned}
$$

which implies that $r(A) \leq\|A-B\|$, that is,

$$
r(A) \leq \inf \left\{\|A-B\|: \overline{R(B)} \subset_{\neq} R(A), N(B) \supset N(A), B \in L(X, Y)\right\}
$$

holds. Conversely, let $x_{n} \in X$ be such that $\left\|x_{n}\right\|=1$ and $\left\|A x_{n}\right\| \rightarrow r(A)$. Then by the Hahn-Banach theorem, there exist $x_{n} \in X^{*}$ such that $x_{n}^{*}\left(x_{n}\right)=\left\|x_{n}\right\|=1$ and $\left\|x_{n}^{*}\right\|=1$.

Let $\left\{B_{n}\right\}$ be a sequence of operators which are defined by $B_{n} x=A x-\left(A x_{n}\right) x_{n}^{*}(x)$ (for $x \in X$ ); then we can prove that $B_{n} \in L(X, Y)$ and $\overline{R\left(B_{n}\right)} \subset_{\neq} R(A)$. In fact, $\left\|B_{n}\right\| \leq 2\|A\|$. Since $N(A)=0$, it follows that $R\left(A^{*}\right)=X^{*}$ and then for an arbitrary integer $n$ there exists $y_{n}^{*} \in Y^{*}$ such that $A^{*} y_{n}^{*}=x_{n}^{*}$. Since

$$
\begin{aligned}
\left(B_{n}^{*} y_{n}^{*}\right) x & =y_{n}^{*}\left(B_{n} x\right)=y_{n}^{*}(A x)-x_{n}^{*}(x) y_{n}^{*}\left(A x_{n}\right) \\
& =\left(A^{*} y_{n}^{*}\right) x-x_{n}^{*}(x) A^{*} y_{n}^{*}\left(x_{n}\right) \\
& =x_{n}^{*}(x)-x_{n}^{*}(x) x_{n}^{*}\left(x_{n}\right)=x_{n}^{*}(x)-x_{n}^{*}(x)=0,
\end{aligned}
$$

we have $y_{n}^{*} \in N\left(B_{n}^{*}\right), y_{n}^{*} \notin N\left(A^{*}\right)$, which implies that $N\left(A^{*}\right) \subset_{\neq} N\left(B_{n}^{*}\right)$. So we have $\overline{R\left(B_{n}\right)} \subset_{\neq} R(A)$. From

$$
\left\|\left(A-B_{n}\right) x\right\|=\left\|A x_{n} x_{n}^{*}(x)\right\| \leq\left\|A x_{n}\right\|\|x\|,
$$

for each $x \in X$, we obtain

$$
\inf \left\{\|A-B\|: \overline{R(B)} \subset_{\neq} R(A)\right\} \leq\left\|A-B_{n}\right\| \leq\left\|A x_{n}\right\| \rightarrow r(A) .
$$


(ii) Suppose that $N(A) \neq 0$. We define a bounded linear operator $\hat{A}$ from $X / N(A)$ to $Y$ by $\hat{A} \hat{x}=A x$, where $\hat{x}$ is the equivalent class of $x$ relative to $N(A)$.

From $N(A) \supset N(B)$, we define $\hat{B} \in L(X / N(A), Y)$ by $\hat{B} \hat{x}=B x$. Since $\overline{R(B)} \subset_{\neq} R(A)$, we have $\overline{R(\hat{B})} \subset_{\neq} R(\hat{A})$. Then by the definition of $\hat{B}$, we obtain

$$
N(B) \supset N(A), \quad \overline{R(B)} \subset_{\neq} R(A) .
$$

In order to prove (ii), we first prove that $\|\hat{A}-\hat{B}\|=\|A-B\|$. For each $y \in N(A)$

$$
\|\hat{A} \hat{x}-\hat{B} \hat{x}\|=\|A x-B x\| \leq\|A(x-y)-B(x-y)\| \leq\|A-B\|\|x-y\| .
$$

Meanwhile, from $\|\hat{x}\|=\inf _{y \in N(A)}\|x-y\| \leq\|x\|$, we have

$$
\|\hat{A} \hat{x}-\hat{B} \hat{x}\| \leq\|A-B\| \inf _{y \in N(A)}\|x-y\|=\|A-B\|\|\hat{x}\|,
$$

which leads to $\|\hat{A}-\hat{B}\| \leq\|A-B\|$.

Conversely, since

$$
\|A x-B x\|=\|\hat{A} \hat{x}-\hat{B} \hat{x}\| \leq\|\hat{A}-\hat{B}\|\|\hat{x}\| \leq\|\hat{A}-\hat{B}\|\|x\|,
$$

we have $\|A-B\| \leq\|\hat{A}-\hat{B}\|$. So we obtain $\|A-B\|=\|\hat{A}-\hat{B}\|$.

Since $N(\hat{A})=0$, by the result of (i) we have

$$
\begin{aligned}
r(A) & =r(\hat{A}) \\
& =\inf \left\{\|\hat{A}-\hat{B}\|: \overline{R(\hat{B})} \subset_{\neq} R(\hat{A}), N(\hat{B}) \supset N(\hat{A}), \hat{B} \in L(X / N(A), Y)\right\} \\
& =\inf \left\{\|A-B\|: \overline{R(B)} \subset_{\neq} R(A), N(B) \supset N(A), B \in L(X, Y)\right\} .
\end{aligned}
$$

COROLLARY 2.4. If $A \in C^{m, n}$, then

$$
r(A)=\inf \left\{\|A-B\|: \operatorname{rank}(A)>\operatorname{rank}(B), B \in C^{m, n}\right\} .
$$

PROOF. By Theorem 2.3,

$$
\begin{aligned}
r(A) & =\inf \left\{\|A-B\|: \overline{R(B)} \subset_{\neq} R(A), N(B) \supset N(A), B \in L(X, Y)\right\} \\
& \geq \inf \left\{\|A-B\|: \operatorname{rank}(A)>\operatorname{rank}(B), B \in C^{m, n}\right\} .
\end{aligned}
$$

On the other hand, by the proof of [3, Lemma 2.3] we have

$$
r(A) \sup \{\operatorname{dist}(u, R(B)): u \in R(A),\|u\|=1\} \leq\|A-B\| .
$$

But from [7, IV-Corollary 3.2], $\sup \{\operatorname{dist}(u, R(B)): u \in R(A),\|u\|=1\}=1$ when $\operatorname{rank} A>\operatorname{rank} B$. Thus we obtain the assertion. 
The following corollary gives another expression of the GCN of a bounded linear operator.

Corollary 2.5. Let $A \in L(X, Y)$, then

$$
\bar{\kappa}(A)=\sup \left\{\frac{\|A\|}{\|A-B\|}: \overline{R(B)} \subset_{\neq} R(A), N(B) \supset N(A), B \in L(X, Y)\right\} .
$$

COROLLARY 2.6. Let $A \in C^{m, n}$, then

$$
\kappa(A)=\sup \left\{\frac{\|A\|}{\|A-B\|}: \operatorname{rank}(A)>\operatorname{rank}(B), B \in C^{m, n}\right\} .
$$

Set $A x=y$ be a linear system, and consider the solution of the perturbed problem

$$
A \hat{x}=y+r
$$

If $A$ is invertible or there exists a generalized inverse $A^{+}$of $A$, then we know that the upper and lower error bounds between the accurate solution $x$ and the perturbed solution $\hat{x}$ are relative to the condition number $\kappa(A)=\|A\|\left\|A^{-1}\right\|$ (if $A$ is invertible) or $\kappa^{+}(A)=\|A\|\left\|A^{+}\right\|$(if $A^{+}$exists). The following theorem is the natural extension of the classical error analysis problem to the case with a more general assumption.

THEOREM 2.7. Let $A \in L(X, Y)$, and $R(A)$ be closed. Let $y \in R(A)$, and $S=$ $\{x: A x=y, y \neq 0\}$. If $\hat{x}$ is a solution of the perturbed problem $A x=y+r$, then

$$
\frac{1}{\bar{\kappa}(A)} \frac{m\|r\|}{\|y\|} \leq \operatorname{dist}(\hat{x}, S) \leq \bar{\kappa}(A) \frac{m\|r\|}{\|y\|},
$$

where $m=\inf _{x \in S}\|x\|, \bar{\kappa}(A)$ is the $\mathrm{GCN}$ defined by (2.2).

PROOF. Let $\left\{x_{n}\right\}_{1}^{\infty} \subset S$ such that $\left\|\hat{x}-x_{n}\right\| \rightarrow \operatorname{dist}(\hat{x}, S)$ as $n \rightarrow \infty$. If $z_{n}=\hat{x}-x_{n}$, then $A z_{n}=A \hat{x}-A x_{n}=y+r-y=r$. From $\|A x\| \geq r(A) \operatorname{dist}(x, N(A))$, we have $\|r\| \geq r(A) \operatorname{dist}\left(z_{n}, N(A)\right)$. Thus

$$
\frac{\|r\|}{r(A)} \geq \inf _{u \in N(A)}\left\|z_{n}-w\right\|=\inf _{w \in N(A)}\left\|\hat{x}-x_{n}-w\right\| \geq \operatorname{dist}(\hat{x}, S)
$$

since $x_{n}+w \in S$. For each $x \in S, A x=y$, we have $\|y\| \leq\|A\|\|x\| \leq\|y\| m$. So we obtain

$$
\frac{\operatorname{dist}(\hat{x}, S)}{m} \leq r(A)^{-1} \frac{\|r\|}{m} \leq \bar{\kappa}(A) \frac{\|r\|}{\|y\|}
$$


Now, we prove the reverse inequality. If $x \in S$, and $z \in N(A)$, we have $x-z \in S$. Then $m \leq\|x-z\|$, which leads to $m \leq \operatorname{dist}(x, N(A))$. Thus, we have

$$
\|A x\| \geq r(A) \operatorname{dist}(x, N(A)) \geq r(A) m .
$$

Since $r=A\left(\hat{x}-x_{n}\right)$ and

so we obtain

$$
\frac{\operatorname{dist}(\hat{x}, S)}{m}=\lim _{n \rightarrow \infty} \frac{\left\|\hat{x}-x_{n}\right\|}{m} \geq \frac{\|r\|}{m\|A\|} \geq \frac{\|r\|}{\kappa(A)\|y\|},
$$

$$
\frac{1}{\bar{k}(A)} \frac{m\|r\|}{\|y\|} \leq \operatorname{dist}(\hat{x}, S) \leq \bar{\kappa}(A) \frac{m\|r\|}{\|y\|} .
$$

\section{The generalized inverse and the GCN}

In this section, we introduce another form of GCN, which is related to an inner inverse (or $\{1\}$-inverse) of $A$. If $A \in L(X, Y)$, an operator $B \in L(Y, X)$ which satisfies $A B A=A$ is called an inner inverse (or $\{1\}$-inverse) of $A$. We denote an inner inverse of $A$ by $A^{-}$.

LEMMA 3.1. If $A \in L(X, Y)$, then $A^{-}$exists if and only if $R(A)$ is closed and both $N(A)$ and $\overline{R(A)}$ have complementary subspaces in $X$ and $Y$, respectively.

Proof. Only if: Let $B \in L(Y, X)$ be such that $A B A=A$. If $x_{n} \in R(A)$ are such that $x_{n} \rightarrow x_{0}$, then there exists $y_{n} \in X$ such that $x_{n}=A y_{n}$. So we have

$$
A B x_{n}=A y_{n} \rightarrow A B x_{0}, \quad \text { that is, } x_{0}=A B x_{0},
$$

which implies that $R(A)$ is closed. Let $P=A B$ and $Q=B A$. Then $P^{2}=P$ and $Q^{2}=Q$. It is easily seen that $P$ and $Q$ are projections from $Y$ and $X$ onto $R(A)$ and $N(A)$ respectively. Thus we conclude that complementary subspaces of $N(A)$ and $\overline{R(A)}$ exist.

If: Let $P$ and $Q$ be projections from $Y$ and $X$ onto $R(A)$ and $N(A)$, respectively. Then $\left.A\right|_{(I-Q) X}$ is an invertible operator from $(I-Q) X$ to $P Y$. We define $B \in L(Y, X)$ by

$$
\begin{cases}B y=\left(\left.A\right|_{(I-Q) X}\right)^{-1} y & \text { if } y \in P y \\ B y=0 & \text { if } y \in(I-P) Y\end{cases}
$$

then $A B A=A$. 
DEFINITION 3.2. If an inner inverse $A^{-} \in L(Y, X)$ of $A \in L(X, Y)$ exist, then

$$
\hat{\kappa}(A)=\|A\| \inf \{\|B\|: A B A=A, B \in L(Y, X)\}
$$

is called the intrinsic condition number (or ICN).

REMARK. (1) If $A$ is invertible, then $\hat{\kappa}(A)=\|A\|\left\|A^{-1}\right\|=\kappa(A)$;

(2) Let $X=H_{1}$ and $Y=H_{2}$ be Hilbert spaces, then we have that $\hat{\kappa}(A)=$ $\|A\|\left\|A^{+}\right\|=\kappa^{+}(A)$.

Now, we present the relation between the generalized condition number and the intrinsic condition number.

THEOREM 3.3. Suppose that the inner inverse $A^{-}$of $A \in L(X, Y)$ exists. Then

$$
\bar{\kappa}(A) \leq \hat{\kappa}(A) .
$$

Proof. From $A B A=A$, we have $\|A x\|=\|A B A x\| \leq\|A\|\|B\|\|A x\|$. For each $x \in X,\|x\| \geq \operatorname{dist}(x, N(A))$, we have

$$
\frac{\|A x\|}{\|x\|} \leq\|A\|\|B\| \frac{\|A x\|}{\operatorname{dist}(x, N(A))}, \quad \text { (if } x \neq 0 \text { ). }
$$

Then $\|A\| \leq\|A\|\|B\| r(A)$, which implies $\bar{\kappa}(A) \leq \hat{\kappa}(A)$.

DEFINITION 3.4. Let $X$ be a Banach space, and $V$ be a complemented subspace in $X$. Then $\pi(V,\|\cdot\|)=\inf \{\|P\|: P$ is the projection operator from $X$ onto $V\}$ is called the projection constant of $V$ with respect to the norm $\|\cdot\|$.

THEOREM 3.5. If an inner inverse $A^{-}$of $A \in L(X, Y)$ exists, then

$$
\max \left\{\bar{\kappa}(A), \pi\left(N(A)^{c},\|\cdot\|_{X}\right)\right\} \leq \hat{\kappa}(A) \leq \bar{\kappa}(A) \pi\left(R(A),\|\cdot\|_{Y}\right) \pi\left(N(A)^{c},\|\cdot\|_{X}\right),
$$

where $N(A)^{c}$ is a complementary subspace of $N(A)$ in $X$.

Proof. Suppose that $B$ is an inner inverse of $A$, that is, $A B A=A$. Define $Q=B A$, which is the projection operator from $X$ onto $N(A)^{c}$ along $N(A)$. Now, $\|Q\| \leq\|B A\| \leq\|B\|\|A\|$. Hence, taking the infimum over all inner inverses of $A$, we get

$$
\pi\left(N(A)^{c},\|\cdot\|_{X}\right) \leq \hat{\kappa}(A) .
$$

From (3.1), we establish the lower bound. 
Now, we prove the reverse inequality. Define $P=A B$, which is a projection operator from $Y$ onto $R(A)$. Then, $\left.P A\right|_{Q X}$ is an invertible operator from $Q X$ onto $P Y$. Define $B_{1}=\left(\left.P A\right|_{Q X}\right)^{-1} P$, then we obtain $B_{1} P=Q B_{1}=B_{1}, B_{1} A=Q$, and $A B_{1}=P$. So we have

$$
\begin{aligned}
\|P\|\|x\| & \geq\|P x\|=\left\|A B_{1} x\right\| \geq r(A) \operatorname{dist}\left(B_{1} x, N(A)\right) \\
& \geq r(A) \frac{1}{\|Q\|}\left\|Q B_{1} x\right\| \geq r(A) \frac{1}{\|Q\|}\left\|B_{1} x\right\|,
\end{aligned}
$$

that is, $\|B\| \leq\|P\|\|Q\| / r(A)$, or $\|A\|\left\|B_{1}\right\| \leq\|A\| r(A)^{-1}\|P\|\|Q\|$, which yields $\hat{\kappa}(A) \leq \bar{\kappa}(A) \pi\left(R(A),\|\cdot\|_{Y}\right) \pi\left(N(A)^{c},\|\cdot\|_{X}\right)$.

COROLLARY 3.6. If $A \in C^{m, n}$, then

$$
\max \left\{\bar{\kappa}(A), \pi\left(R\left(A^{*}\right),\|\cdot\|_{D}\right)\right\} \leq \hat{\kappa}(A) \leq \bar{\kappa}(A) \pi\left(R\left(A^{*}\right),\|\cdot\|_{D}\right) \pi(R(A),\|\cdot\|),
$$

where $\|\cdot\|_{D}$ is the dual norm of $\|\cdot\|$.

The above corollary presents a more general result than that of Proposition 3 in [4].

At the end, we consider when $\bar{\kappa}(A)=\hat{\kappa}(A)$. First, we have the following lemma (see [5]).

LEMMA 3.7. Let $\mu, \nu$ be measures, and $T: L_{p}(\nu) \rightarrow L_{p}(\mu)(p \geq 1$ and $p \neq 2)$ be an isometric embedding. Then there exists a projection operator $P$ from $L_{p}(\mu)$ onto $R(T)$ such that $\|P\| \leq 1$.

By Lemma 3.7 and Theorem 3.5, we have

PROPOSITION 3.8. Let $\mu, \nu$ be measures, and $A: L_{p}(\nu) \rightarrow L_{p}(\mu)$ be a bounded linear operator. Let also $R(A)$ be closed, and $N(A)=0$. If $p \geq 1$ and $p \neq 2$, and $\|A x\|=\|x\|$ for each $x \in L_{p}(\nu)$, then $\bar{\kappa}_{p}(A)=\hat{\kappa}_{p}(A)$.

COROLLARY 3.9. Let $A \in C^{m \times n}$ and $N(A)=0$. If $\|A x\|=\|x\|$ for each $x \in C^{n}$, then $\bar{\kappa}_{p}(A)=\hat{\kappa}_{p}(A)$, where $1 \leq p \leq \infty$ and $p \neq 2$.

\section{Acknowledgements}

The first author was supported by the National Natural Science Foundation of China under grant 19871029 and Shanghai Priority Academic Discipline. The second author was supported by the National Natural Science Foundation of China under grant 19901006 and China Scholarship Council. The third author was supported 
by the National Natural Science Foundation of China under grant 10071020 and Fundamental Research Fund of ECUST.

The authors are grateful to the referee and editors for kindly pointing out some errors in the paper.

\section{References}

[1] G. Chen, 'Minimum property in some problems with condition number of square matrices', Comm. Appl. Math. Comput. 1 (1988), 74-80 (Chinese),

[2] G. Chen and D. Chen, 'On the minimum property of condition number for doubly perturbed linear equations', J. Math. Applicata 3 (1989), 69-76 (Chinese).

[3] G. Chen and Y. Xue, 'Perturbation analysis for the operator equation $T x=b$ in Banach spaces', J. Math. Anal. Appl. 212 (1997), 107-125.

[4] S. Demko, 'Condition numbers of rectangular systems and bounds for generalized inverses', Linear Algebra Appl. 78 (1986), 199-206.

[5] L. E. Dor, 'On projections in $L_{1}$ ', Ann. of Math. (2) 102 (1975), 463-474.

[6] N. Dunford and J. T. Schwartz, Linear operators. Part 3: Spectral operators (Wiley, New York, 1971).

[7] T. Kato, Perturbation theory for linear operators, 2nd edition (Springer, Berlin, 1984).

[8] J. Kuang, 'The $\omega$-condition number of linear operators', Numer. Math. J. Chinese Univ. 1 (1980), 11-18 (Chinese).

[9] J. R. Pragua, 'New condition number for matrices and linear systems', Computing 41 (1989), 211-213.

[10] A. E. Taylor, Introduction to functional analysis (Wiley, New York, 1958).

[11] H. Wolkowicz and S. Zlobec, 'Calculating the best approximate solution of an operator equation', Math. Comp. 32 (1978), 1183-1213.

[12] Y. Xue, 'The reduced minimum modulus of elements in $C^{*}$-algebras', preprint.

Department of Mathematics

East China Normal University

Shanghai 200062

P.R. China

Department of Mathematics

East China University of

Science and Technology

Shanghai 200237

P.R. China

e-mail: xyf63071@public9.sta.net.cn
Department of Mathematics

Fudan University

Shanghai 200433

P.R. China 